\title{
LE POLITICHE DELLA SCUOLA IN EUROPA. UN'ANALISI COMPARATA
}

\author{
di Sofia Ventura
}

\section{Premessa}

Le politiche dell'istruzione costituiscono un ambito di studio rispetto al quale la scienza politica, nonché la più specifica disciplina dell'analisi delle politiche pubbliche, presentano notevoli lacune.

$\mathrm{Da}$ un lato, nella letteratura internazionale le numerose ricerche di education policy si sono concentrate prevalentemente su studi del caso singolo e hanno dedicato minore attenzione all'analisi comparata delle politiche scolastiche ${ }^{1}$. Le quali, inoltre, sono state assai poco esplorate nell'ambito dei più generali studi comparati dedicati al welfare state, che hanno piuttosto privilegiato lo studio delle politiche relative ai sistemi di assicurazione sociale; come ha rilevato Alber: «la sola ricerca empiricamente consolidata» in tale settore (Alber 1987, 15).

Dall'altro, nella letteratura politologica italiana la politica scolastica costituisce un terreno pressoché inesplorato ${ }^{2}$. In Italia essa è stata, infatti, dominio dei pedagogisti, che hanno privilegiato approcci di tipo normativo, dei filosofi e, in misura minore, degli storici, il prevalente taglio descrittivo dei quali non ha, però, favorito lo sviluppo di analisi di tipo esplicativo (Benadusi 1989a, 258; Trivellato 1989, 226).

1 Tra le più rilevanti eccezioni ricordiamo i lavori di Arnold J. Heidenheimer e lo studio della sociologa inglese Margareth Archer (1984). Nella letteratura italiana l'unico tentativo di proporre un'analisi delle politiche scolastiche in chiave comparata è quello compiuto dal sociologo dell'educazione Luciano Benadusi (1989a; 1989b).

2 Oltre ai lavori di Benadusi sono da segnalare, tra le poche eccezioni, quelli di Trivellato $(1984 ; 1989)$ per il caso italiano e la recente pubblicazione di Gianfranco Rescalli (1996), che ha proposto un'analisi dei processi di riforma degli ordinamenti scolastici in cinque democrazie europee: Inghilterra, Svezia, Spagna, Francia e Germania.

RIVISTA ITALIANA DI SCIENZA POLITICA / a. XXVII, n. 2, agosto 1997 
La scarsa attenzione può non meravigliare se si guarda alla limitata salienza che questa politica ha avuto nel nostro paese sia presso l'opinione pubblica, sia presso i policymakers. Essa, però, appare grave se si considera l'importanza delle istituzioni scolastiche per i rispettivi contesti politici, sociali ed economici ${ }^{3}$.

Non si può dimenticare come la genesi dei sistemi pubblici di insegnamento sia strettamente legata ai processi di formazione dello Stato e della nazione e, dunque, all'affermazione ed estensione dei diritti di cittadinanza. Inoltre, negli ultimi decenni, tali sistemi sono stati percepiti dalle élite politiche, sociali, economiche e culturali del mondo occidentale come importanti strumenti per il mantenimento o la trasformazione degli assetti sociali esistenti, in virtù della loro funzione di socializzazione delle nuove generazioni. Così come sono stati ritenuti fattori essenziali per lo sviluppo economico, nel contesto di un sistema produttivo e di una società che esigono una sempre più elevata preparazione culturale.

Affermazione della cittadinanza politica e sociale, democratizzazione, sviluppo economico, sono tutti fenomeni rispetto ai quali il funzionamento dei sistemi scolastici è stato considerato un importante elemento propulsivo. Da qui la rilevanza delle scelte pubbliche in tale settore. E dunque, a nostro avviso, l'importanza di studi che siano in grado sia di descrivere, sia di proporre chiavi esplicative dei processi di policy relativi.

Con questo saggio intendiamo fornire un contributo in tale direzione, attraverso l'analisi dei processi di riforma che hanno investito gli ordinamenti scolastici europei a livello secondario, a partire dagli anni sessanta, e dei potenziali fattori esplicativi di tali processi e dei relativi esiti.

Per quanto concerne tali fattori, metteremo a fuoco con particolare attenzione le caratteristiche politico-istituzionali dei sistemi politici, nonché quelle dei sistemi di amministrazione e governo dell'istruzione, elementi che l'analisi da noi condotta ha rilevato essere in grado di facilitare o inibire l'innovazione in istituzioni come quelle scolastiche, caratterizzate da un'alta $v i$ schiosità.

Tali innovazioni, come è noto, sono inserite nell'agenda

3 Un'interessante teoria sullo stretto legame esistente tra strutture educative e sistema sociale è stata elaborata da John W. Meyer (1977). 
dell'attuale governo italiano. Ci pare, dunque, che una riflessione su alcuni dei più rilevanti fattori che possono condizionare le trasformazioni del sistema educativo costituisca un interessante punto di partenza per analizzare e valutare il processo di policy che si sta avviando nel nostro paese per introdurre importanti trasformazioni nell'ordinamento della scuola secondaria superiore e nel più generale sistema di amministrazione della scuola.

Infine, la rilevanza dello specifico processo di riforma, la trasformazione degli ordinamenti della scuola secondaria, da noi analizzato, deriva, da un lato dalla sua diffusione in quasi tutti i paesi europei, dall'altro dal fatto che esso ha costituito un tentativo di risposta ad uno dei più rilevanti fenomeni sociali del secondo dopoguerra, vale a dire l'affermazione dell'insegnamento secondario come insegnamento di massa.

Se l'istruzione primaria aveva assunto caratteri di massa già alla fine del secolo scorso, infatti, è solo dopo la seconda guerra mondiale che l'istruzione secondaria assume progressivamente la connotazione di una istruzione per tutti.

$\grave{E}$, infatti, a partire dagli anni cinquanta, e con maggiore intensità dagli anni sessanta, che $\mathrm{i}$ sistemi scolastici europei vengono investiti da un verticale aumento della domanda di scolarizzazione al livello secondario. Questa esplosione della domanda di scolarizzazione, sempre più percepita come strumento di mobilità sociale, si accompagna in quegli anni ad una fase di espansione dell'economia e allo sviluppo di «retoriche educazionali» e di «climi di opinione» (Benadusi 1989a) favorevoli ad una democratizzazione dell'insegnamento secondario. Democratizzazione intesa come strumento per realizzare l'eguaglianza delle opportunità educative, in un contesto di scolarizzazione di massa nel quale la semplice diffusione dell'offerta educativa non è più considerata strumento sufficiente per realizzare tale obiettivo, vale a dire per eliminare i condizionamenti sociali sul percorso e sul successo scolastico degli studenti. In questo periodo, inoltre, notevole consenso riscuote, presso le élite intellettuali come presso le classi dirigenti europee, la cosiddetta «teoria del capitale umano», che individua nella formazione scolastica un fattore di impulso per lo sviluppo economico.

A queste pressioni di carattere economico, sociale e culturale, seguiranno importanti trasformazioni nei sistemi scolastici di gran parte dei paesi europei. Trasformazioni che verranno introdotte principalmente negli anni sessanta e nei primi anni set- 
tanta e che coinvolgeranno in primo luogo l'ordinamento degli studi.

Le riforme attuate dai governi europei durante questa prima ondata di riforme vanno nella direzione del superamento, più o meno radicale, della tradizionale tripartizione degli studi secondari in scuole accademiche, tecniche e generali (scuole postprimarie generalmente senza alcuno sbocco) diffusa, pur con differenze da un caso all'altro, in tutta Europa. Queste trasformazioni, inoltre, si accompagnano sovente ad un innalzamento del limite della scuola dell'obbligo, che al termine di questo periodo avrà raggiunto quasi ovunque il sedicesimo anno di età $\mathrm{e}$ in taluni casi anche il diciottesimo (Germania e Belgio).

$\mathrm{Ma}$ ancor più, le trasformazioni della scuola secondaria permettono una più chiara definizione degli ambiti di quella primaria, che giunge definitivamente ad assumere chiaramente il ruolo di scuola elementare, dove cioè vengono insegnati i primi elementi delle diverse discipline, intesa come primo grado degli studi, unico per tutti e obbligatorio. Non come un settore separato, la cosiddetta scuola per il popolo, rispetto a quello secondario, frequentato da chi è destinato a proseguire gli studi e comprendente proprie classi elementari.

Questa prima ondata di riforme è stata seguita da una seconda ondata, a partire dagli anni ottanta, quando molti paesi cominciano a mettere in discussione le strutture dei loro sistemi di insegnamento e, quindi, la capacità di queste di rispondere alle nuove sfide, poste innanzitutto dalle trasformazioni del mercato del lavoro e in particolare dall'evoluzione scientifica e tecnologica da un lato, e dal carattere strutturale assunto dalla disoccupazione, specie quella giovanile, anche dopo la ripresa economica seguita alla crisi innestata dallo shock petrolifero del 1973, dall'altro. In tale contesto vengono elaborati e posti in essere progetti di riforma in numerosi ambiti dell'insegnamento. Le trasformazioni indotte da questo secondo periodo di riforme, ancora non concluso, hanno però coinvolto in misura marginale, a parte alcune eccezioni, la struttura dell'ordinamento scolastico.

Poiché il nostro obiettivo è quello di mettere a fuoco i fattori che hanno favorito o inibito le trasformazioni degli ordinamenti scolastici secondari ereditati dal secolo scorso nei diversi casi nazionali, dedicheremo esclusiva attenzione alla prima fase delle riforme, e prenderemo in considerazione interventi ad essa 
successivi solo laddove essi riguardino direttamente la configurazione dell'ordinamento scolastico.

Accentramento e decentramento nei sistemi di amministrazione dell'istruzione

Prima di procedere all'analisi delle principali riforme attuate nel contesto europeo, sarà utile soffermarci brevemente sulle caratteristiche dei sistemi di governo e amministrazione dell'istruzione, in quanto proprio tali caratteristiche rientrano tra $\mathrm{i}$ potenziali fattori esplicativi degli esiti delle riforme nei paesi qui considerati.

A questo proposito va rilevato che la semplice, e diffusa, distinzione tra sistemi accentrati e decentrati di governo dell'istruzione riduce la complessità delle possibili conformazioni delle strutture concernenti l'insegnamento, tanto più se si adotta una prospettiva di analisi di tipo diacronico ${ }^{4}$. Ai nostri fini adotteremo qui una distinzione tra quei sistemi che, nel periodo delle principali riforme della scuola secondaria, mantengono importanti prerogative a livello nazionale, in particolare per quanto concerne l'organizzazione dell'ordinamento degli studi, e quelli che invece hanno demandato ad altri livelli tali prerogative.

Nella prima classe rientrano paesi quali la Francia, l'Italia, la Spagna, la Svezia. Nella seconda la Gran Bretagna, il Belgio, l'Olanda e la Germania.

Il sistema scolastico della Francia deriva il proprio carattere accentrato dalla politica scolastica di Napoleone I, avviata nei primissimi anni del secolo scorso e mirante alla costituzione di un sistema di istruzione pubblico sottoposto, in tutti i suoi gradi, al rigido controllo dell'Università, organizzata localmente in Académies, ciascuna con a capo un rettore, e con competenze assai vaste (Prost 1968, 23). I numerosi regimi che da allora si sono succeduti non hanno messo in discussione tale assetto, ma lo hanno piuttosto perfezionato, in particolare durante gli anni della Terza Repubblica. Ancora oggi esso si presenta con una struttura piramidale e gerarchica, con al proprio vertice il Ministère de l'Education Nationale. Le competenze dello Stato in

4 Importanti cambiamenti sono stati infatti introdotti in numerosi paesi, in particolare negli anni ottanta e novanta. 
materia di educazione sono state tradizionalmente molto ampie, andando dalla definizione degli obiettivi pedagogici e dei programmi alla concessione dei titoli di studio; dal reclutamento, gestione e retribuzione del personale all'organizzazione territoriale dell'offerta di istruzione nei suoi diversi gradi (Benadusi 1989a; Ocde 1996). Solo negli anni ottanta e novanta è stato avviato un parziale processo di decentramento dell'amministrazione scolastica, a favore, in particolare, dei livelli regionale, provinciale (départements) e comunale dell'amministrazione medesima. Processo che non ha però intaccato il ruolo centrale dello Stato nell'educaziones.

In Italia l'amministrazione scolastica è stata forgiata sul modello napoleonico. Nel periodo repubblicano l'impianto generale ereditato dai precedenti regimi, e di carattere fortemente accentrato, è rimasto sostanzialmente invariato. $\grave{E}$ stato anzi pienamente recepito l'apporto dato dal fascismo alla sua espansio$n e^{6}$. Le parziali innovazioni introdotte negli anni settanta, con la creazione del ministero dei Beni culturali che ha limitato l'azione di quello dell'istruzione al solo ambito dell'insegnamento, e con la creazione delle regioni, alle quali sono state demandate alcune marginali competenze in materia scolastica ${ }^{7}$, non hanno inciso sul tradizionale assetto centralizzato dell'amministrazione scolastica italiana (Gigante 1988).

Per tutto il periodo precedente la costituzione del 1978, in Spagna il governo del sistema pubblico di istruzione ha mantenuto un carattere fortemente accentrato, con una struttura am-

5 L'amministrazione centrale francese ha infatti conservato le responsabilità legate ai principi di uguaglianza e laicità e al carattere di servizio pubblico dell'insegnamento, responsabilità che si concretizzano nella definizione degli obiettivi generali delle politiche educative; nella definizione degli orientamenti pedagogici e dei contenuti dell'insegnamento, nella regolamentazione dei titoli e delle modalità per il loro ottenimento; nella formazione, remunerazione e gestione del personale insegnante e non insegnante dell'istruzione secondaria (Ocde 1996, 35).

6 Come è stato a tale proposito rilevato: «il modello di amministrazione scolastica che sulla base del ruolo predominante assunto dall'intervento statale in materia di istruzione si era venuto affermando nell'epoca giolittiana, e che il fascismo aveva non solo tenuto fermo, ma anzi consolidato, risulta ora confermato, ed ulteriormente ampliato, negli anni del dopoguerra» (Gigante 1988, 187).

7 Con la creazione delle regioni l'azione statale è stata limitata ai soli interventi formativi, mentre alle regioni sono stati attribuiti gli interventi strumentali al servizio scolastico vero e proprio, come l'edilizia e l'assistenza scolastica, nonché quegli interventi formativi di carattere professionale o comunque non diretti all'acquisizione di un titolo. Si tratta di materie per le quali lo Stato ha comunque mantenuto poteri di indirizzo e coordinamento. 
ministrativa ruotante attorno al Ministero dell'Educazione e della Scienza e articolata a livello centrale in Direzioni generali e sul territorio in Delegazioni provinciali. Con la divisione dei compiti amministrativi tra Stato, comunità ed enti locali sancita dalla Costituzione del 1978, anche l'amministrazione scolastica ha subito un importante processo di decentramento, a favore, in particolare, delle comunità autonome. Lo Stato ha però mantenuto importanti responsabilità, riguardanti la regolamentazione dei titoli accademici e professionali, la definizione dei principi generali dell'educazione, la definizione degli ordinamenti generali del sistema di istruzione, la fissazione dei punti fondamentali relativi agli insegnamenti minimi e ai curricoli di base comuni a tutto il territorio dello Stato (Rescalli 1996).

Meno centralizzato appare il sistema di amministrazione scolastica della Svezia, caratterizzato da una sostanziale diarchia tra poteri centrali e autonomie locali (Ocde 1981, 71) «a cavallo tra centralismo e autonomie, fortemente decentrato a livello locale ma saldamente controllato e governato dall'Amministrazione centrale» (Rescalli 1996, 79). A livello nazionale hanno infatti, fino ad anni recenti, svolto un ruolo centrale per l'organizzazione e le trasformazioni del sistema scolastico il Parlamento, le commissioni governative e il Consiglio nazionale scolastico, sorto nel 1920 e soppresso nel 1990, organo preposto all'amministrazione e alla gestione dell'istruzione primaria e secondaria. Gli interventi degli anni ottanta volti all'accentuazione dell'autonomia delle autorità locali non hanno messo in discussione il tradizionale equilibrio tra centro e periferia e la capacità di controllo del livello nazionale. Equilibrio che pare sia stato messo in discussione solo in seguito alle più radicali trasformazioni degli anni novanta (Rescalli 1996).

La carenza di un forte controllo a livello centrale dell'amministrazione scolastica contraddistingue, o ha contraddistinto fino ad anni recenti, gli altri quattro paesi da noi analizzati, paesi che presentano però una elevata eterogeneità rispetto al tipo di decentramento in essi posto in essere.

Per quanto riguarda la Gran Bretagna, o per meglio dire l'Inghilterra e il Galles, fino alle riforme introdotte a partire dal 1988, che hanno condotto ad un notevole rafforzamento del livello centrale e dell'autonomia dei singoli istituti scolastici, il governo del sistema di istruzione ha mantenuto il proprio fulcro nelle Local Education Authorities (Lea), istituite nel 1902 e le cui competenze sono state ridefinite con l'Education Act del 
1944. Competenze che hanno riguardato non solo l'ambito amministrativo, ma anche quello relativo alla definizione delle linee generali dei curricoli e dell'area comune di insegnamento, nonché l'organizzazione dell'offerta formativa (ad es. chiusura ed apertura di scuole) nel territorio di propria competenza. All'interno di tale sistema di pluralismo diffuso un ruolo importante è sempre stato attribuito alle singole unità scolastiche, competenti per la dimensione culturale degli studi (definizione dei programmi e contenuti delle materie).

In questo contesto, le prerogative del livello nazionale sono state limitate a compiti di indirizzo e distribuzione dei fondi. A tale proposito è però importante rilevare come, a partire dalla seconda metà degli anni sessanta, il governo centrale abbia assunto una maggiore iniziativa rispetto alle scelte compiute dalle singole autorità locali nell'ambito dell'ordinamento degli studi (Fenwick e Mc Bride 1981; Rescalli 1996).

Profondamente diverso è il decentramento che caratterizza il governo dell'istruzione proprio della Germania. In tale caso, infatti, vi è una attribuzione pressoché esclusiva, sanzionata dall'articolo 31 della Legge fondamentale dell'8 maggio 1949, delle competenze legislative e amministrative in materia culturale ed educativa ai Länder. Tra le poche eccezioni previste vi sono le specifiche competenze del governo federale in materia di istruzione professionale, di concessione di borse di studio, di sovvenzioni alla ricerca scientifica e di regolamentazione dell'accesso alla carriera insegnante. L'esistenza di strutture formali di collegamento tra gli stati e tra questi e il governo federale non è stata sufficiente ad imprimere un indirizzo unitario alla politica scolastica tedesca. Come mostra la fallimentare esperienza della «Commissione federazione-Länder per la programmazione dell'educazione e la promozione della ricerca», istituita nel 1970 e soppressa nel 1983, e del «Consiglio nazionale dell'insegnamento» (1965-1975). E come mostrano, ancora, le difficoltà incontrate dal più importante di tali organismi, la «Conferenza permanente dei ministri dell'Educazione e degli Affari culturali», nata nel 1949 per coordinare le politiche scolastiche dei diversi stati. Fin dall'inizio, infatti, le prerogative di tale organismo sono state limitate dalla regola dell'unanimità vigente per tutte le decisioni da prendere al suo interno, nonché dal fatto che tali decisioni hanno sempre avuto, dal punto di vista giuridico, il carattere di semplici raccomandazioni, non vincolanti se non fatte proprie dai governi e dai legislatori dei singoli stati. 
È inoltre importante osservare come il decentramento si fermi al livello statale. I singoli stati hanno infatti sviluppato al loro interno altrettanti sistemi di governo dell'istruzione altamente centralizzati. Si è perciò parlato, per il caso tedesco, di un sistema multicentralizzato, profondamente differente da quello di pluralismo diffuso proprio del caso inglese (MaxPlanck-Institute 1991; Rescalli 1996).

Originale appare infine la struttura dell'amministrazione scolastica in Olanda e in Belgio. In entrambi i paesi accanto al sistema pubblico di istruzione, vi è una forte presenza di istituzioni educative private, prevalentemente confessionali (cattoliche in Belgio; cattoliche e protestanti in Olanda), che godono di ampia autonomia, scolarizzano circa il $70 \%$ della popolazione scolastica, e che sono poste su un sostanziale piano di parità, dal punto di vista dei finanziamenti statali, con quelle pubbliche.

In Belgio la costituzione del 1831 e poi il Pacte scolaire del 1959 , stipulato tra le diverse forze politiche del paese, hanno riconosciuto il principio della libertà di insegnamento e la possibilità di gestione del medesimo ad una molteplicità di istituzioni: lo Stato, le province, i comuni, le associazioni di comuni, i privati e gli enti religiosi (Draghicchio 1980, 139). Il governo dell'istruzione poggia dunque su una molteplicità di pouvoirs organizateurs che gestiscono le scuole di propria competenza con ampia autonomia organizzativa e didattica (Bdp 1994, 6) e il sistema nel suo complesso appare rigidamente suddiviso in una molteplicità di reseaux ufficiali (statali, provinciali e comunali) e non ufficiali (insegnamento privato). La complessità del sistema è stata poi ulteriormente rafforzata dalla divisione linguistica che ha condotto, nel 1988, alla attribuzione delle competenze statali alle comunità culturali francofona, fiamminga $\mathrm{e}$ germanofona.

Nel sistema dell'Olanda sono individuabili quattro tipi principali di scuole: le scuole pubbliche, le scuole private non confessionali, le scuole private cattoliche e quelle protestanti. I gruppi con interessi legati al sistema di istruzione (ad es. le associazioni dei genitori e i sindacati degli insegnanti) si sono sviluppati separatamente, all'interno dei quattro settori che compongono il sistema di insegnamento, e afferiscono ad altrettante organizzazioni ombrello che rappresentano e tutelano gli interessi dei diversi tipi di scuole presso gli organismi consultivi che collaborano all'attività del Ministero della Scienza. Organismi che costi- 
tuiscono, dunque, i luoghi di negoziazione e consultazione tra il Ministro e le diverse parti in causa, tra i quali si svolgono regolari incontri, coerentemente con la tradizione consociativa olandese (Ocde 1991, 121-124). Inoltre, se le possibilità di intervento del governo sono maggiori nel settore pubblico che in quello privato, anche al di fuori di quest'ultimo le scuole olandesi godono di ampia autonomia. Le limitate prerogative del governo centrale, inoltre, vengono sempre gestite attraverso le consultazioni delle organizzazioni di vertice dell'insegnamento.

\section{Verso l'unitarietà degli studi secondari}

All'interno del contesto europeo abbiamo individuato otto casi nazionali, che si differenziano per il diverso livello di unitarietà raggiunto dai rispettivi ordinamenti secondari: Belgio, Francia, Germania, Gran Bretagna, Italia, Olanda, Spagna e Svezia.

Seguendo Jean-Michel Leclercq (1993) possiamo distinguere i paesi europei in tre diverse classi, in base al tipo di riforme che hanno attuato in questo campo. La prima classe riguarda quei paesi che non hanno apportato sostanziali modifiche alla ripartizione tradizionale tra scuole classiche, tecniche e generali e che mantengono un sistema caratterizzato da una precoce selezione (undici anni). L'unico paese tra quelli da noi presi in considerazione che ricade in questa classe è la Germania, affiancata da altri tre paesi europei: la Svizzera, il Lussemburgo e l'Austria. La seconda classe contiene invece quei paesi che hanno sì apportato mutamenti, ma che non sono giunti alla creazione di una scuola unitaria, limitandosi, in taluni casi, all'aggregazione di più percorsi nelle stesse strutture e al rinvio della formazione professionale al livello di studi post-obbligatorio. Due sono i casi che vi rientrano: il Belgio e l'Olanda. La terza classe è, infine, rappresentata da quei paesi che hanno completamente superato la tradizionale ripartizione a canne d'organo e che hanno dato vita ad una scuola unitaria almeno al livello secondario inferiore. Ad essa appartengono la Svezia, la Francia, la Spagna, la Gran Bretagna, l'Italia e altri paesi in questa sede non analizzati quali la Grecia, il Portogallo e la Danimarca.

Nell'ambito europeo la Svezia e la Germania rappresentano le due esperienze più diverse relativamente alle politiche di integrazione della scuola secondaria: «Il tentativo di comparare le 
iniziative di riforma adottate in Svezia e in Germania federale è stimolante poiché i due paesi si collocano agli estremi della graduatoria europea in materia di unitarietà degli studi secondari» (Heidenheimer 1974, 388).

I governi socialdemocratici della Svezia hanno realizzato in un trentennio un modello di scuola unica di base di nove anni, dai 7 ai 16 anni, obbligatoria (la Grundskola), seguita da una scuola secondaria unitaria ma articolata al suo interno in un numero molto elevato di «linee», della durata variabile da due a quattro anni (Gymnasieskolan) (Zocchi 1989, 27-28). Precedentemente il sistema svedese era caratterizzato da due canali paralleli. Un primo canale, non selettivo, era rappresentato dalla scuola primaria, obbligatoria dai sette ai quattordici anni, gestito dalle autorità comunali e che permetteva l'eventuale proseguimento degli studi nelle cosiddette scuole di prosecuzione, in scuole professionali o altri tipi di scuola secondaria inferiore. Un secondo canale, al quale si accedeva dopo quattro anni di scuola elementare, era invece gestito direttamente dallo Stato ed era composto da un livello inferiore, la Realskola (di 4 o 5 anni), che dava l'accesso al Gymnasium (di 3 o 4 anni) e quindi agli studi universitari.

Le richieste di una maggiore eguaglianza nelle possibilità di accesso alla scuola secondaria e dunque per la creazione di una scuola comprensiva e unificata erano già state avanzate all'inizio del secolo dai movimenti popolari particolarmente attivi nella società svedese ed in particolare dal movimento sindacale e dalle organizzazioni degli insegnanti ad esso legate (Husén 1986, 155). Ma fu nel 1948 che per la prima volta, da una commissione governativa, emerse la proposta di creare una scuola unica di nove anni. Un proposta che divenne legge nel 1950, quando il Parlamento approvò il provvedimento con il quale si sarebbe dato il via ad una diffusa sperimentazione, che alla fine degli anni cinquanta, era arrivata a coinvolgere un quarto della popolazione (Boucher 1982, 27-29; Husén 1986, 158). In questo processo il ruolo degli esperti fu di notevole rilievo, anche se, come è stato osservato, la decisione finale di estendere il modello della scuola di base di nove anni obbligatoriamente a tutto il territorio con la legge di riforma del 1962, fu principalmente una decisione politica (Boucher 1982, 30).

Con la legge del 1962 fu istituita la Grundskola, con la quale furono riuniti i precedenti livelli elementare e secondario inferiore, una scuola di base completamente indifferenziata al suo 
interno e articolata in tre diversi gradi, dai 7 ai 10 , dai 10 ai 13 , dai 13 ai 16 anni.

Anche la creazione della nuova scuola secondaria è avvenuta per tappe successive. Già nel 1953 alle sezioni moderna e classica del ginnasio era stata aggiunta una sezione scientifica. Nel 1961 le tre sezioni del Ginnasio generale furono accorpate con il Ginnasio tecnico e il Ginnasio commerciale, andando così a formare una scuola superiore unitaria, articolata al suo interno in cinque linee di tre anni ciascuna (quattro per la linea tecnica). Con la legge del 1966 furono riformate anche le scuole para-professionali e professionali, che con la legge di riforma del 1971 sarebbero andate a confluire nella nuova scuola secondaria (Gymnasieskolan), una scuola comprensiva articolata in linee di diversa durata e in una moltitudine di corsi specialistici (Boucher 1982, 34-37; Zocchi 1989, 5-6).

Nonostante anche la Germania sia stata investita dal movimento riformatore degli anni sessanta e settanta, essa ha mantenuto sostanzialmente immutate le caratteristiche fondamentali dell'ordinamento degli studi, riorganizzato dopo la seconda guerra mondiale durante l'occupazione degli Alleati e con le proprie radici nel secolo XIX. Oggi il sistema tedesco, che si sta progressivamente estendendo ai Länder orientali, si distingue per due caratteristiche originali rispetto al più generale panorama europeo: la elevata selettività dell'organizzazione degli studi e la centralità dell'insegnamento professionale. La durata del ciclo elementare è di soli quattro anni (sei a Berlino), al termine dei quali gli scolari vengono selezionati, con procedure diverse tra $\mathrm{i}$ vari stati, per essere inseriti nei tre differenti percorsi della scuola secondaria: il Gymnasium, della durata di nove anni (sei di livello inferiore e tre di livello superiore) e che costituisce l'unica via per raggiungere l'insegnamento universitario; la Realschule, che fornisce una preparazione tecnico-scientifica, della durata di sei anni, che permette l'accesso alla formazione tecnica o professionale di livello secondario superiore (anche a tempo parziale) di due o tre anni e quindi all'istruzione superiore non universitaria, anch'essa di carattere tecnicoprofessionale; la Hauptschule, della durata di cinque anni, che costituisce il percorso meno prestigioso ed ha lo scopo di fornire la base dei corsi di formazione professionale successivi, corsi che però non danno l'accesso all'istruzione superiore non universitaria. Nel sistema tedesco è presente anche la Gesamtschule, scuola secondaria inferiore unitaria, che permette l'ac- 
cesso ai tre anni del secondo ciclo del Gymnasium, ma che scolarizza soltanto circa il 5\% degli studenti tedeschi (Max-PlanckInstitute 1991; Rescalli 1996) ${ }^{8}$.

L'altra particolarità del sistema tedesco è costituita dall'attenzione dedicata alla formazione professionale, che ha inizio solo al livello superiore della scuola secondaria. Essa presenta un'ampia articolazione ed anche la possibilità di accedere (per alcuni percorsi) agli studi superiori non-universitari fino al livello corrispondente al dottorato del settore universitario.

Il sistema, così come si configura oggi, è, nelle sue linee essenziali, il prodotto delle parziali innovazioni introdotte negli anni sessanta e settanta nel sistema riorganizzato al termine della seconda guerra mondiale. La fase delle innovazioni ha inizio, nella Repubblica Federale Tedesca, alla fine degli anni cinquanta e si conclude all'inizio degli anni settanta (Rescalli 1996, 236237). Il primo tentativo di riforma risale all'importante piano del 1959: il Rabmenplan. Questo (Piano per la ristrutturazione e l'unificazione del sistema pubblico di istruzione generale) $\mathrm{fu}$ elaborato da un organo governativo di carattere consultivo, il «Comitato nazionale per l'educazione e l'istruzione». Il progetto, che a causa dei limitati poteri attribuiti al comitato altro non poteva avere che il carattere di raccomandazione, non metteva in discussione la conformazione tripartita dell'ordinamento degli studi e proponeva l'introduzione di un ciclo di orientamento di due anni (Förderstufe), successivo ai quattro anni del corso elementare, con l'obiettivo di ridurre la selezione precoce degli allievi (Pasini 1977, 254). Esso garantiva però la preservazione del corso di nove anni (10-19 anni) del Gymnasium, prevedendo la possibilità per gli studenti più capaci di evitare tale periodo di orientamento e di accedere direttamente al percorso accademico (Enderwitz 1963, 48-49). Il piano fu seguito, nel 1964, dall'accordo di Amburgo tra i ministri dell'istruzione dei Länder. I principali punti di tale accordo riguardarono la gene-

8 E però importante rilevare che tra questi diversi canali esiste una certa potenziale mobilità, ad esempio nelle Hauptschule agli studenti meritevoli al quinto anno è rilasciato un certificato che permette l'iscrizione negli altri canali. Al tempo stesso all'interno sia della Hauptschule, sia del Gymnasium è possibile ottenere un diploma equivalente a quello rilasciato dalla Realschule. Questi passaggi, però, riguardano una esigua percentuale di studenti, così come quantitativamente marginale è anche la cosiddetta «seconda via dell'istruzione», costituita da istituti a tempo parziale o a tempo pieno che, principalmente con corsi serali, permette di conseguire diplomi di maturità generale o specializzata (Franchi e Segantini 1994, 68; Rescalli 1996, 207). 
ralizzazione dell'obbligo scolastico al nono anno di scolarità; l'invito rivolto ai diversi Länder a generalizzare i due anni di orientamento; e lo spostamento al decimo anno di scolarità della netta differenziazione degli indirizzi del Gymnasium, in modo tale da permettere l'accesso agli ultimi tre anni di questo agli studenti diplomati alla Realscbule.

Per quanto attiene la sperimentazione di una scuola unitaria, la Conferenza dei ministri si rifiutò di renderla obbligatoria per tutto il territorio. A partire dall'accordo di Amburgo cominciò comunque una sperimentazione in tal senso. Nel 1969, anno in cui va al governo la coalizione tra socialdemocratici e liberali, il Consiglio nazionale dell'insegnamento raccomanda l'istituzione sperimentale di quaranta istituti di scuole unitarie (Gesamtschule). Lo stesso anno la Conferenza dei ministri raggiunge un accordo su un programma sperimentale che prevede due varianti di Gesamtscchule, quella integrata, che comporta la totale soppressione dei tre canali, e quella cooperativa, che si limita invece a farli coesistere sotto lo stesso tetto (Max-PlanckInstitute 1991, 202). È proprio però sull'istituzione della $G e$ samtschule che le politiche degli stati cominceranno a differenziarsi notevolmente. I più ampi tentativi di ristrutturazione del sistema in questo senso sono infatti stati condotti dagli stati governati dai socialdemocratici, mentre le tendenze più conservatrici si sono registrate in quelli a maggioranza democratico-cristiana. Questo ha creato notevoli scompensi territoriali ed ha anche inibito il processo di innovazione, impedendo ai Länder più progressisti di procedere troppo oltre nelle riforme a causa del problema del riconoscimento dei titoli rilasciati nelle scuole del nuovo tipo nelle altre parti del paese (Pasini 1977, 259260). Non meraviglia dunque il sostanziale fallimento dell'esperienza della Gesamtschule.

Più ambizioso sul piano teorico fu il Piano strutturale per il sistema educativo del 1970, elaborato dal Consiglio nazionale tedesco, che, tra gli altri obiettivi, si era posto quelli della generalizzazione e obbligatorietà del ciclo di orientamento; l'unificazione del primo ciclo della scuola secondaria; e la graduale unificazione di tutti i canali della scuola secondaria, compresi i cicli terminali. Un piano ambizioso che fu accantonato dalla Conferenza dei Ministri. Il piano proposto, infine, dalla Commissione per la pianificazione educativa (il luogo di concertazione tra stati e governo federale) nel 1973, costituì un realistico arretramento rispetto alle proposte del 1970 . Esso infatti si limitò a re- 
gistrare il dissenso tra gli stati sull'unificazione del primo ciclo della scuola secondaria rinunciando a dare indicazioni operative e abbandonando così al «destino di una sperimentazione senza fine e affidata di fatto alla volontà dei Länden» la Gesamtscbule (Rescalli 1996, 246).

Oltre alla Svezia i paesi che hanno introdotto riforme orientate alla creazione di un percorso unitario al livello di istruzione secondaria sono, come abbiamo visto, l'Italia, la Francia, la Spagna e la Gran Bretagna.

Per quanto riguarda l'Italia, sarà qui sufficiente ricordare che le trasformazioni in senso unitario dell'ordinamento scolastico hanno coinvolto esclusivamente il livello secondario inferiore. Con la legge del 1962 viene infatti varata la scuola media unica. Una scuola di tre anni con la quale venivano soppressi i precedenti canali, risalenti alla riforma Bottai del 1940, della scuola media, dell'avviamento e dei corsi di avviamento professionale. Per quanto concerne il livello secondario superiore, a partire dagli anni settanta e fino agli anni novanta, sono stati fatti numerosi tentativi di introdurre una organica riforma dell'ordinamento, tentativi che sono però sempre falliti, anche a causa dell'impossibilità di trovare un accordo tra i diversi partiti politici, in particolare tra la Dc da un lato e il Pci e il Psi dall'altro, coinvolti nel processo decisionale, un processo estremamente diffuso e comprendente le forze politiche sia della maggioranza che dell'opposizione.

Anche nel caso della Francia l'unitarietà è stata realizzata solo nella scuola secondaria inferiore, anche se, a differenza dell'Italia, importanti riforme hanno investito anche il livello superiore. Il sistema scolastico francese prevede una scuola elementare di sei anni, a partire dal sesto anno di età, seguita da un livello secondario inferiore organizzato in un percorso unitario di quattro anni (collège), che prevede però alcune opzioni che relativizzano tale unitarietà. Se infatti i primi due anni (sixième e cinquième - ciclo di osservazione) sono comuni, è possibile, da un lato uscire dal collège dopo la quinta classe per intraprendere una via di carattere professionale senza sbocchi sull'insegnamento superiore, dall'altro inserirsi, negli ultimi due anni (quatrième e troisième), in un percorso a carattere tecnologico, sempre all'interno del collège, che permette una prosecuzione verso studi di carattere professionale anche a livello di insegnamento superiore (Fanelli 1989, 132-135; Ocde 1996, 29-30). Una particolarità del sistema francese è inoltre data dal sistema di orien- 
tamento degli alunni al termine della quinta classe (questo snodo è stato eliminato nel 1989) e della terza classe per l'accesso all'istruzione secondaria superiore. Un sistema formalizzato nel 1973 e sostanzialmente basato sulle decisioni prese dal Consiglio di classe, sentiti gli orientamenti dei genitori, su proposta del Consiglio dei professori (Fanelli 1989, 135-136; Prost 1992, 155-156).

Il processo di trasformazione del tradizionale ordinamento degli studi sviluppatosi durante gli anni sessanta e giunto ad una prima conclusione con la legge Haby del 1975, si è svolto in maniera frammentaria, a causa anche delle resistenze presenti in importanti settori dell'amministrazione scolastica (Prost 1992, 88).

Le prime critiche al tradizionale sistema di insegnamento francese risalgono al secondo decennio del secolo, anche se i primi timidi cambiamenti al suo interno saranno realizzati solo negli anni trenta. Ciò che prima di tutto veniva contestato al sistema di insegnamento francese, in particolare da parte dei partiti della sinistra (socialisti e radicali) ma anche da organizzazioni storicamente legate all'insegnamento pubblico (come la Ligue de l'enseignement), era il parallelismo esistente tra l'insegnamento primario e quello secondario. La scuola per il popolo, primaria, si distingueva nettamente da quella secondaria, per la borghesia, e tra le due non vi era alcun collegamento. I primi limitati interventi su questa struttura furono avviati, tra la fine degli anni venti e gli anni trenta, durante la permanenza al ministero dell'istruzione del radicale Herriot e successivamente del ministro del Fronte popolare Jean Zay. Quest'ultimo intervenne con alcune misure regolamentari che ebbero come unico, ma non irrilevante, effetto, di integrare le scuole primarie superiori (Eps) - ma non i corsi complementari (Cc) post-elementari, privi di ulteriori sbocchi - al settore secondario. Una integrazione che avrebbe trovato una più radicale realizzazione nel 1941, durante il governo di Vichy, ad opera del ministro Carcopino che attuò la trasformazione delle Eps in collegi moderni, che permettevano l'accesso alla sezione moderna dei licei e dunque al baccalauréat. Il tema dell'école unique, intesa come scuola media unica per tutti, doveva poi essere affrontato nel noto piano Langevin-Wallon del 1947, che, come ogni altro tentativo e progetto della Quarta repubblica, non ebbe alcun seguito. Sarà soltanto negli anni della Quinta repubblica, con una coalizione di governo di centro-destra e con alla presidenza 
della Repubblica il generale Charles de Gaulle (che si impegnò direttamente nei progetti di trasformazione del sistema scolastico), che progressive e importanti riforme del sistema verranno realizzate.

Il passo decisivo verso il superamento del dualismo si ha con la riforma del 1963, che prende il nome dall'allora ministro Fouchet ma che deve la sua realizzazione principalmente alla volontà del presidente De Gaulle. Con tale riforma le classi dei collèges dell'insegnamento secondario (Sixième, cinquième, quatrième e troisième), spesso attaccate ai licei, vengono separate da questi (che progressivamente si riducono così a tre classi, deuxième, première e terminale) con la creazione di istituti specifici, i Ces, collèges d'enseignement secondaire. I collèges continuano ad essere organizzati sia nel settore primario $(\mathrm{Ceg})$, sia in quello secondario (Ces). Le differenze tra i due, però, vanno progressivamente assottigliandosi grazie alla creazione di nuove sezioni all'interno di entrambi che li rendono sempre più simili e che costringono alla compresenza nei due diversi tipi di collegio di insegnanti provenienti dal settore primario e da quello secondario. A questo punto appare ormai irreversibile la riorganizzazione del sistema per gradi e non per comparti separati. La riforma Haby (legge dell'11 luglio 1975 e decreti del 28 dicembre 1976) costituisce il punto di arrivo di questa evoluzione. Essa abolisce ogni distinzione tra Ceg e Ces e pone fine ad ogni differenziazione in sezioni nei primi due anni del collège (ciclo di osservazione), mentre nei due successivi (ciclo di orientamento) permangono scelte opzionali accanto all'insegnamento comune che preparano alle scelte successive e sono dunque orientative (Prost 1968, 405-426; Prost 1992, 68-95; Fanelli 1989, 92-95).

La Spagna costituisce un caso originale rispetto a quelli qui analizzati per il fatto che essa si è trasformata in un regime democratico solo dopo il 1975, con la fine del regime autoritario franchista. A questo ci pare debba essere addebitato il fatto che il provvedimento legislativo che ha realizzato una scuola unitaria in due gradi, elementare e secondario inferiore, fino all'età di sedici anni, sia di recente approvazione, risalendo soltanto al 1990. La prima importante trasformazione in senso unitario risale però agli ultimi anni del regime franchista, gli anni più liberaleggianti, ed è del giugno 1970 .

L'organizzazione degli studi posta in essere dalla legge generale sull'educazione del 1970 comporta una radicale revisione 
rispetto al sistema precedente. Quest'ultimo prevedeva infatti una scuola primaria di otto anni, gli ultimi quattro anni della quale erano paralleli all'insegnamento secondario di primo grado, al quale si accedeva, con un esame, dopo i primi quattro anni dell'insegnamento primario, e costituiva un percorso seguito da una esile minoranza (Draghicchio 1980, 397; Leclercq $1993,31)$. Con la riforma del 1970, che pone l'obbligo scolastico a quattordici anni, viene creato un insegnamento generale di base unitario di otto anni (Egb), dai sei ai quattordici anni, che incorpora il ciclo elementare e il primo ciclo secondario. La scuola secondaria di secondo grado della durata di tre anni, inoltre, viene unificata nel Bachillerato unificado polivalente, una scuola unitaria articolata in materie comuni e materie opzionali che, dopo un ulteriore anno di orientamento universitario (Cou, curso de orientacion universitaria), permette l'accesso all'istruzione superiore sia universitaria, sia non universitaria. Parallelamente al Bachillerato, però, è previsto un corso professionale organizzato in due cicli biennali, separato dall'insegnamento generale (Rescalli 1996, 89). La legge del 1970 e quella del 1990, che modifica ulteriormente l'organizzazione degli studi, sono separate da una fase di grave recessione economica, comune agli altri paesi europei, che ha inizio con la crisi petrolifera del 1973. Una fase alla quale corrisponde, nel campo scolastico, una flessione nell'impegno finanziario dello Stato e durante la quale viene addirittura messa in dubbio la validità degli obiettivi fondamentali della riforma del 1970. La ripresa economica che ha luogo a partire dagli anni ottanta, accompagnata da importanti conquiste sul piano della democratizzazione delle strutture sociali, registra una importante inversione di tendenza. Il dibattito sulla scuola riprende, collocandosi all'interno del più generale dibattito sulle riforme, e viene portato avanti, sul piano politico, con particolare vigore dal partito socialista spagnolo (Psoe), al governo dal 1982. In questo contesto, infatti, la spinta riformatrice non proviene tanto dalle forze economiche, come era avvenuto negli ultimi anni del regime autoritario, ma piuttosto dall'ambito politico ed in particolare dai settori di punta del Psoe, in questa sua azione riformatrice osteggiato principalmente dalla Chiesa cattolica spagnola e dall'Ucd, il partito di centro-destra di ispirazione cattolica. La riforma del 1990, preceduta dalla legge organica sul diritto all'educazione (Lode) che ha esteso l'obbligatorietà della frequenza scolastica ai sedici anni, è seguita ad un breve periodo (1983-1987) di 
sperimentazioni riguardanti l'unificazione del biennio nella fascia di età che va dai quattordici ai sedici anni, nonché da un dibattito nazionale avviato dal ministro dell'educazione Maravall nel 1987 su un progetto di riforma globale del sistema di insegnamento elaborato dal Consiglio educativo centrale (istituito con la legge del 1985).

Il nuovo modello scaturito dalla «legge di orientamento generale del sistema scolastico» scinde la formazione di base di otto anni, poiché prevede un insegnamento elementare di sei anni seguito da un insegnamento secondario inferiore di quattro anni, obbligatorio, diviso in due bienni successivi, unitario ma caratterizzato da un curricolo flessibile con materie obbligatorie e materie opzionali (Franchi e Segantini 1994, 58-59). La nuova organizzazione dell'insegnamento comporta dunque uno spostamento della scelta tra percorso generale e percorso professionale a sedici anni. All'interno della scolarità obbligatoria non è dunque prevista alcuna formazione professionale. Inoltre gli stessi canali generale e professionale dell'insegnamento secondario superiore sono stati profondamente rinnovati.

L'ultimo tra i paesi da noi analizzati che ha introdotto tra gli anni sessanta e settanta trasformazioni nell'ordinamento dell'insegnamento secondario in senso unitario (trasformazioni che hanno coinvolto anche il livello secondario superiore) è l'Inghilterra (con il Galles). Attualmente il sistema prevede un insegnamento obbligatorio dai cinque ai sedici anni, da ottemperarsi nelle scuole elementari, primary schools, e in quelle secondarie inferiori. Il livello secondario inferiore ha la durata di cinque anni (11-16). L'offerta quantitativamente preponderante a questo livello è fornita dalla comprebensive school, la scuola unitaria che scolarizza circa il $90 \%$ della popolazione scolastica di quella fascia, accanto alla quale permangono le grammar schools (175 scuole, secondo dati recenti, contro le 1180 del 1965), le modern schools (da 3498 scuole nel 1965 a 285) e le technical schools (in via di estinzione) (Rescalli 1996, 22). Le grammar schools, sorte nel XVI secolo, sono tradizionalmente le più prestigiose del sistema inglese e forniscono una istruzione di taglio prevalentemente classico. Esse ricoprono l'intero livello secondario, scolarizzando studenti che vanno dagli undici ai diciotto anni. Le technical schools forniscono invece una preparazione di tipo tecnico, mentre le modern schools (eredi delle elementary senior schools) impartiscono una preparazione a carattere generale di tipo breve e pratico, per studenti che per lo più non 
proseguono gli studi. Entrambe le scuole hanno durata quinquennale (Morini 1977, 292-293; Giunta La Spada 1989, 122 123; Leclercq 1993, 25). Gli studi della fascia post-obbligo possono poi proseguire nel livello superiore delle comprebensive schools o nel cosiddetto sixth form college (un ciclo biennale di preparazione all'esame di maturità) (Giunta La Spada 1989, 24) $\mathrm{o}$, per coloro che provengono dai primi cinque anni della grammar school, nei restanti due anni finali della medesima. I diplomi non hanno alcun valore legale e sono le singole Università che fissano in maniera autonoma i requisiti di accesso.

Nell'immediato dopoguerra il sistema scolastico inglese presentava un'organizzazione degli studi basata sulla classica tripartizione che abbiamo già incontrato in altri paesi.

L'Education Act del 1944, però, seguendo le indicazioni della commissione Speen del 1938, che proponeva di riorganizzare la scuola secondaria in tre settori separati (grammar school, technical school e modern school), e della commissione Norwood del 1941, che fornì un sostegno pedagogico a tale proposta (Rescalli 1996, 4), nell'attribuzione dei compiti educativi alle Lea stabilì che le scuole dovessero essere

per numero, carattere e mezzi, idonee a consentire a tutti gli alunni di fruire di quelle varietà di educazione e di formazione specifica che meglio risponde alle diversità di età, di attitudini, e dei differenti periodi di frequenza scolastica richiesti dagli alunni stessi (cit. in Morini 1977, 293).

L'interpretazione di questa clausola, date anche le prevalenti tendenze pedagogiche dell'epoca, dominanti anche nel partito laburista, da parte della maggioranza delle Lea non poteva che condurre ad una sostanziale adesione al sistema tripartito, che avrebbe raggiunto la sua massima espansione negli anni quaranta e cinquanta (Rescalli 1996, 4-5).

La legge del 1944 non costituendo un vincolo tassativo per le Lea per quanto riguarda l'organizzazione delle scuole, permise comunque ad alcune di esse, tra cui quella di Londra, di avviare la sperimentazione delle comprebensive schools. Alcuni importanti fattori favorevoli allo sviluppo della scuola in senso unitario, emersero, inoltre, negli anni cinquanta. Da un lato vi fu l'adozione del modello comprensivo da parte del partito laburista. Dall'altra il prodursi di importanti pressioni sulla scuola da parte dei ceti sociali emergenti nel dopoguerra. Essi, collocati tra la classe operaia e la classe media tradizionale, desiderosi di stabilizzare la propria posizione sociale, avevano comin- 
ciato ad esercitare una domanda crescente di istruzione nelle grammar schools. A causa della ristrettezza dei posti disponibili, ben presto le pressioni di tali ceti furono indirizzate verso la creazione di una scuola secondaria che superasse il sistema tripartito e assorbisse le grammar schools. Non a caso dunque i leader laburisti cominciarono a diffondere il loro programma educativo riferendosi ad una «grammar school for all».

Dopo i primi esperimenti di scuole comprensive negli anni quaranta, l'esperienza cominciò a diffondersi in un numero sempre più ampio di Lea (comprese alcune controllate dai conservatori, che fino al 1951 si erano mostrati favorevoli a limitati esperimenti in aree scarsamente popolate). Una diffusione alla quale non furono estranee le numerose ricerche pedagogiche che demolirono i risultati delle commissioni Speen e Norwood e misero in luce l'importanza delle discriminazioni sociali all'interno del sistema selettivo; ricerche che favorirono l'adesione al modello comprensivo di quelle Lea laburiste ancora riluttanti ad adottarlo. La progressiva diffusione del quale assunse un carattere impetuoso negli anni sessanta (Rescalli 1996, 6-10). Fino a quel momento, però, gli interventi sull'organizzazione degli studi secondari erano stati promossi pressoché esclusivamente dalle Lea. Fu, infatti, solo nel 1965 che si giunse ad un riconoscimento governativo della comprebensive school, quando il nuovo ministro laburista dell'Educazione, Anthony Crosland, emanò una circolare nella quale si chiedeva alle Lea di elaborare e sottoporre dei piani per la riorganizzazione dell'istruzione secondaria, nelle zone di loro competenza, su «direttrici di tipo unitario» (Rescalli 1996, 10; Morini 1977, 295). A questa prima circolare seguì quella del governo conservatore nel 1970, con la quale il ministro dell'Educazione Margareth Thatcher rimosse le indicazioni precedenti, indicazioni che sarebbero state infine ribadite in una terza circolare nel 1974 dal governo laburista tornato al potere. Soltanto nel 1976 il partito laburista si decise ad affrontare la questione con un intervento legislativo che ponesse fine alla situazione di incertezza. Con la legge del 1976, infatti, il Parlamento attribuì al segretario di Stato per l'educazione il potere istituzionale di attuare su tutto il territorio la scuola secondaria unitaria, affidando alle Lea il compito di realizzare le trasformazioni richieste sul piano strutturale e amministrativo. L'approvazione della legge giunse però in un periodo caratterizzato dall'emergere di numerose critiche alla qualità dell'insegnamento impartito nel Regno Unito, critiche che pro- 
venivano sì da parte conservatrice ma che ben presto furono fatte proprie dallo stesso partito laburista. Ebbe inizio così una inversione di tendenza, rispetto al clima che aveva accompagnato lo sviluppo delle comprebensive schools, che si collocava nel contesto della recessione mondiale cominciata nel 1973 e di un mutato atteggiamento dell'opinione pubblica inglese rispetto al nuovo sistema scolastico. Una inversione di tendenza che sarebbe stata accentuata dalla vittoria dei conservatori nel 1979, i quali, come primo provvedimento nel campo dell'istruzione, emanarono una legge per revocare l'obbligo fatto alle Lea nel 1976 di ristrutturare in senso comprensivo la scuola secondaria (Rescalli 1996, 11-14). Tali mutamenti non sarebbero però stati tali da mettere in discussione l'ormai affermato sistema delle comprebensive schools.

Il Belgio e l'Olanda costituiscono due esempi di innovazioni parziali nel settore dell'organizzazione degli studi secondari e si collocano perciò in una situazione intermedia rispetto al caso tedesco, da un lato, e a quelli svedese, italiano, francese, spagnolo e inglese, dall'altro.

Il sistema del Belgio è stato rinnovato in seguito alla legge del 19 luglio 1971. Il sistema tradizionale belga, che in parte continua a sussistere, prevede una scuola primaria di sei anni, al termine della quale gli studenti vengono orientati verso tre diverse sezioni: la sezione generale, la sezione tecnica e la sezione professionale, della durata di tre anni. La prima, suddivisa al suo interno in una sezione prettamente classica ed in una moderna, conduce agli studi liceali, della durata di tre anni (anch'essi differenziati); la seconda e la terza permettono invece di proseguire gli studi rispettivamente in istituti tecnici e professionali (questi ultimi senza sbocco sugli studi superiori), anch'essi di durata triennale (Leclercq 1993, 17; Di Francia e Luggeri 1991, 35-37).

Già a partire dal dopoguerra si erano moltiplicate le prese di posizione a favore di un'apertura del sistema, in particolare da parte del partito socialista. Solo nel 1971, però, fu approvato il provvedimento legislativo con il quale fu rinnovata parzialmente la scuola secondaria. In seguito ad esso l'insegnamento rinnovato è stato reso obbligatorio per le scuole dipendenti dallo Stato e consigliato agli altri reseaux. Esso prevede, dopo i sei anni di scuola elementare, tre cicli biennali, un primo di osservazione, un secondo di orientamento ed un terzo di specializzazione (determination). Gli ultimi due cicli sono divisi vertical- 
mente in due settori: la sezione di transizione, finalizzata all'accesso agli studi superiori (universitari e non); la sezione di qualificazione, finalizzata principalmente all'entrata nel mondo del lavoro, anche se permette il proseguimento degli studi. Il primo ciclo biennale è invece un ciclo comune, anche se prevede una struttura differenziata per taluni alunni. A tale livello, infatti, accanto al primo anno «A», è previsto un primo anno «B» al quale sono indirizzati gli scolari che hanno raggiunto risultati non considerati soddisfacenti durante il corso elementare. A seconda dei progressi realizzati, questi possono poi essere indirizzati o verso il secondo anno comune, o verso il secondo anno professionale.

Nel decennio successivo alla riforma, l'insegnamento secondario rinnovato si è generalizzato velocemente nel settore statale. Nella regione francese sia la rete di istituti provinciali, sia quella di istituti cattolici si è adeguata al nuovo modello, mentre più restie a farlo si sono mostrate le scuole comunali. Nel settore fiammingo, invece, mentre i due terzi delle scuole comunali nel 1980 avevano già aderito al nuovo modello, alla stessa data solo il $20 \%$ di quelle provinciali e il $10 \%$ di quelle private (cattoliche) avevano fatto altrettanto. Questa situazione ha condotto nel 1990 la comunità fiamminga ad imporre, per realizzare una organizzazione dell'insegnamento più omogenea, a tutti i reseaux un quadro generale molto simile al modello rinnovato per quanto riguarda il primo biennio, ma più differenziato rispetto ai bienni successivi. Nelle comunità francese e tedesca, invece, a quella data il sistema tradizionale era pressoché estinto (Ocde 1992, 56). Questi processi sono stati accompagnati, in Belgio, da notevoli controversie che hanno contrapposto «i difensori degli ideali di democratizzazione e di equità a coloro che temevano l'appiattimento che il nuovo dispositivo avrebbe potuto determinare» (Ocde 1992, 58). Ed è proprio il permanere di tali contrapposizioni, insieme alle difficoltà finanziarie, che ha contribuito a rendere lenta la realizzazione della riforma e che ha condotto, negli ultimi anni, ad adottare misure, come quella della comunità fiamminga, che fanno temere un ritorno alla situazione precedente (Ocde 1992, 58). In sintonia con queste osservazioni è quanto rileva Leclercq a proposito delle ragioni che hanno consentito la relativa diffusione del nuovo modello, da ricercare a suo avviso nel fatto che l'insegnamento rinnovato si è, quasi fin dall'inizio, allontanato dalle modalità di funzionamento inizialmente previste, come dimostra il fatto che 
all'inizio degli anni novanta il primo anno «B» e il secondo anno professionale scolarizzavano il $25 \%$ del totale degli allievi del primo biennio (Leclercq 1993, 19).

Il sistema dell'Olanda ha conosciuto innovazioni ancora più parziali, rispetto all'obiettivo dell'unitarietà, di quanto non sia avvenuto in Belgio. Il sistema oggi in vigore risale alla legge del 1963, operativa dal 1968, che, insieme alla sucessiva legge sull'insegnamento professionale del 1966, anch'essa effettiva dal 1968 , ha messo ordine nella complessa organizzazione dell'insegnamento che risaliva al 1903 (Leclercq 1993, 20; Ocde 1994, 14-15). Il sistema precedente prevedeva l'insegnamento obbligatorio fino a quattordici anni. L'obbligo poteva essere ottemperato, dopo i sei anni della scuola elementare, in una serie di istituzioni post-elementari appartenenti però al settore primario (come nel caso francese). Le pressioni per una trasformazione del sistema, particolarmente importanti dopo la seconda guerra mondiale, condussero il governo olandese a presentare, nell'ottobre del 1958, un progetto di riforma dell'insegnamento postelementare. Dopo quattro anni di dibattiti, studi e modifiche il progetto, con l'appoggio dei partiti della coalizione di centrosinistra, fu approvato alla prima Camera e nel 1963 definitivamente alla seconda Camera (Van Lutsemburg Maas 1964, 279. 282). Il sistema scaturito da quella legge, e, come già osservato, da quella successiva sull'insegnamento professionale, è organizzato in un insegnamento elementare che va dai sei ai dodici anni (dal 1985 accorpato all'insegnamento pre-scolastico, obbligatorio dai cinque anni), a partire dal quale si diramano quattro diversi tipi di insegnamento. Il primo di questi è l'insegnamento pre-universitario (Vwo), di sei anni, l'unico che dà accesso agli studi universitari; il secondo è l'insegnamento secondario generale superiore (Havo), di cinque anni, che permette di proseguire gli studi al livello superiore non universitario; il terzo è l'insegnamento secondario generale medio (Mavo), di quattro anni, che permette di proseguire gli studi nell'insegnamento secondario professionale di secondo ciclo (Mbo), della durata variabile tra i due e i quattro anni e che dà l'accesso all'insegnamento superiore di carattere professionale; l'ultimo, infine, è l'insegnamento secondario professionale di primo ciclo (Lbo), di tre anni, che permette di proseguire gli studi nel corso professionale Mbo (Leclercq 1993, 20; Ocde 1991).

Come risulta evidente da questa breve descrizione il sistema secondario olandese ha mantenuto comunque un elevato grado 
di differenziazione al livello inferiore e dunque continua ad essere caratterizzato da una scelta precoce degli studi, pur con i correttivi introdotti per una maggiore vicinanza dei percorsi. Tale sistema è ormai dall'inizio degli anni settanta al centro del dibattito. Un dibattito che però, fino al 1992, non ha condotto ad ulteriori riforme a causa dell'impossibilità di raggiungere un consenso sulla natura dei cambiamenti da avviare attraverso il complesso sistema decisionale olandese poggiante sulla consultazione; ma anche a causa dell'assenza di un sostegno popolare a cambiamenti di struttura. Tale contesto ha portato la coalizione liberale e democratico-cristiana a richiedere al Comitato consultivo della politica governativa (Wrr) di elaborare una proposta di riforma alternativa rispetto alla soppressione delle filières, proposta che è stata presentata nel 1986 (Ocde 1991, 35 ) e che ha condotto all'elaborazione del decreto del ministero dell'Educazione e della Scienza del 1992. Tale decreto ha avviato una sperimentazione, da condurre fino al 1997, i cui contenuti rappresentano l'evidente compromesso tra la necessità di unificare i percorsi e le opposizioni esistenti. L'innovazione consiste nella determinazione di obiettivi comuni in quattordici discipline che tutti gli studenti dovranno affrontare durante la scuola secondaria di primo ciclo, lasciando però la possibilità di accedere ai risultati previsti secondo ritmi variabili. Il tutto, però, all'interno delle strutture esistenti (Leclercq 1993, 21).

\section{Un tentativo di spiegazione}

Al termine di questa breve rassegna delle principali trasformazioni che hanno investito gli ordinamenti scolastici europei, è possibile trarre alcune conclusioni circa i fattori che, nei diversi paesi, ne hanno influenzato gli esiti.

Come abbiamo visto è possibile porre i paesi analizzati in tre diverse classi, utilizzando il criterio dell'unitarietà dei percorsi scolastici secondari come possibile esito delle politiche di riforma. Avremo così una prima classe $(\mathrm{A})$ nella quale rientrano quei paesi (Francia, Gran Bretagna, Italia, Spagna e Svezia) nei quali la prima ondata riformatrice ha condotto alla creazione di percorsi scolastici unitari, al livello secondario inferiore ed eventualmente anche a quello superiore; una seconda classe (B) che comprende i paesi (Belgio e Olanda) che hanno introdotto solo parziali modifiche; una terza classe $(\mathrm{C})$ che individua i pae- 
si (Germania) nei quali non è stata apportata nessuna sostanziale modifica.

La Svezia è il paese che più di altri ha realizzato un insegnamento secondario unitario, con la creazione, nel 1962, di una scuola novennale di basse unitaria e indifferenziata al suo interno, e con la sussunzione in un'unica struttura triennale, la Gymnasienskola, dei differenti canali della scuola secondaria superiore, pur mantenendo all'interno di questa una differenziazione in linee, talmente articolata, però, da rendere solo un ricordo la tradizionale tricotomia. La Gran Bretagna ha realizzato anch'essa una scuola comprensiva, comprendente entrambi i livelli dell'insegnamento secondario. Non ha eliminato però completamente le scuole tradizionali, che hanno comunque assunto un carattere quantitativamente marginale nel complesso del sistema, così come ha mantenuto e sviluppato percorsi professionali (further education) nell'insegnamento secondario superiore. L'Italia ha dato vita, nel 1962, alla scuola media unica, che sostituiva i precedenti percorsi della scuola media, della scuola di avviamento e dei corsi di avviamento professionale. Le trasformazioni hanno però interessato esclusivamente la scuola secondaria inferiore. La Spagna, in seguito alle riforme del 1970 e del 1990 , ha costruito un sistema caratterizzato dall'unitarietà del corso secondario inferiore (12-16 anni), l'uscita dal quale, però, è differenziata sulla base dei risultati conseguiti e può condurre o verso l'istruzione generale, aperta sullo sbocco universitario, unificata nel Bachillerato già dal 1970, o verso l'istruzione professionale. Anche la Francia ha limitato l'unitarietà dell'insegnamento al livello secondario inferiore (anche se la riforma Haby investì parzialmente anche il livello secondario superiore, poi ulteriormente trasformato negli anni ottanta e novanta). Al suo interno, inoltre, essa ha mantenuto la possibilità di uscita al termine del primo biennio del corso quadriennale unitario, nonché una linea orientata agli studi professionali nel secondo biennio. Il sistema francese è inoltre caratterizzato da un severo sistema di orientamento che limita le possibilità di accesso ai diversi corsi secondari superiori, che, nonostante i tentativi di riforma solo parzialmente attuati, presentano una struttura fortemente differenziata, condizionante l'accesso agli studi superiori. Il Belgio e l'Olanda, come abbiamo visto, hanno introdotto riforme molto parziali, rispetto alla dimensione differenziazione/ unitarietà. Nel sistema del Belgio esiste solo un primo biennio comune, che contiene però al suo interno un percorso che pre- 
clude, pur con dei correttivi, la via dell'insegnamento generale (prima classe «B» e seconda professionale). Il successivo biennio e il livello secondario superiore continuano invece ad essere separati in diversi canali. Inoltre continua a sussistere, anche se solo in alcuni ambiti, il sistema tradizionale. L'Olanda ha introdotto una razionalizzazione e semplificazione del sistema mantenendo però la differenziazione in percorsi separati già a partire dall'uscita dell'insegnamento elementare, lasciando dunque a tale livello la selezione. La Germania, infine, ha mantenuto il sistema tripartito ricostituito nel 1949.

L'individuazione dei fattori esplicativi di tali differenze è tutt'altro che semplice, a causa dello stretto legame esistente tra i sistemi di insegnamento e le dimensioni economica, sociale, culturale, politica e, più in generale, storica dei singoli Stati. Tenteremo, comunque, di districare dal groviglio delle specificità nazionali quei fattori che più di altri ci paiono aver condizionato i processi e gli esiti delle politiche scolastiche in questo ambito.

A questo proposito Luciano Benadusi, per spiegare le differenze tra cinque grandi democrazie (la Svezia, la Gran Bretagna, la Francia, l'Italia e la Germania), ha individuato quattro fattori principali: il contesto economico e del welfare, il sistema istituzionale, il sistema politico, il clima politico e le retoriche educazionali prevalenti (Benadusi 1989a, 206). Relativamente al primo fattore ci pare di poter affermare che l'andamento ciclico dell'economia e l'espansione o la contrazione delle politiche di welfare siano fattori in grado di spiegare il generale orientamento delle politiche scolastiche europee ad attuare trasformazioni nei sistemi di insegnamento piuttosto che le differenze. Le diversità nell'andamento dell'economia e le conseguenti possibilità di sviluppare politiche di welfare, inoltre, ci paiono altamente insufficienti per spiegare le diverse politiche. Non si capirebbe perché, altrimenti, ad un più limitato sviluppo del Pil procapite tra il 1960 e il 1980 in Gran Bretagna rispetto alla Francia, siano corrisposte politiche più incisive, rispetto all'obiettivo dell'unitarietà, nel primo paese anziché nel secondo. Ci pare, piuttosto, che l'impatto sui singoli sistemi nazionali del generale andamento dell'economia possa avere costituito un elemento che, all'interno di una complessa configurazione di fattori, abbia reso più o meno praticabile l'adozione di certe politiche, l'origine delle quali va però ricercata in altre dimensioni, diverse da 
TAB. 1. Unitarietà degli studi e grado di accentramento istituzionale

\begin{tabular}{|c|c|c|c|}
\hline & A & B & $\mathrm{C}$ \\
\hline $\begin{array}{l}\text { Sistemi } \\
\text { accentrati }\end{array}$ & $\begin{array}{l}\text { Francia } \\
\text { Italia } \\
\text { Spagna } \\
\text { Svezia }\end{array}$ & & \\
\hline $\begin{array}{l}\text { Sistemi } \\
\text { decentrati }\end{array}$ & Gran Bretagna & $\begin{array}{l}\text { Belgio } \\
\text { Olanda }\end{array}$ & Germania \\
\hline
\end{tabular}

TAB. 2. Unitarietà degli studi e forza decisionale dei governi

\begin{tabular}{llll}
\hline & A & B & C \\
\hline $\begin{array}{lll}\text { Governi } \\
\text { forti }\end{array}$ & $\begin{array}{l}\text { Francia } \\
\text { Gran Bretagna }\end{array}$ & & Germania \\
$\begin{array}{l}\text { Spagna } \\
\text { Svezia }\end{array}$ & & \\
$\begin{array}{l}\text { Governi } \\
\text { deboli }\end{array}$ & Italia & $\begin{array}{l}\text { Belgio } \\
\text { Olanda }\end{array}$ & \\
\hline
\end{tabular}

Dalla tab. 1 emerge un dato interessante: dei cinque paesi che hanno realizzato, anche se in misura diversa, riforme in senso comprensivo, quattro (Francia, Italia, Spagna e Svezia) hanno sistemi di governo dell'istruzione centralizzati. Per quanto concerne la seconda variabile, la tab. 2 pone in evidenza come dei cinque paesi che hanno introdotto riforme in senso unitario, quattro (Francia, Spagna, Svezia e Gran Bretagna) hanno governi forti.

Ciò ci potrebbe condurre a formulare due prime ipotesi sul valore esplicativo di tali variabili. Con la prima potremmo porre una relazione positiva tra accentramento delle istituzioni preposte al governo del sistema scolastico e possibilità di introdurre importanti innovazioni nell'ordinamento degli studi. Con la seconda porre una relazione positiva tra quest'ultimo fattore e l'elevata capacità decisionale dei governi.

Relativamente al primo fattore, l'unica eccezione è costituita dalla Gran Bretagna, che si presenta come un caso nel quale sono state realizzate riforme in senso comprensivo in assenza di interventi vincolanti da parte del governo nazionale. $\mathrm{Ci}$ sembra che il caso inglese indichi la possibilità di una diversa via alle riforme, una via che si snoda attraverso le iniziative di autorità locali di governo del sistema di insegnamento (le Lea) poste al livello immediatamente superiore alle unità scolastiche. Una via 
quella economica, che, ripetiamo, ci pare spiegare più le somiglianze che le differenze.

Le caratteristiche istituzionali, vale a dire la configurazione del sistema di amministrazione e decisione dell'insegnamento, che può assumere diverse conformazioni a seconda della distribuzione delle competenze tra differenti livelli di governo, e le più generali caratteristiche politico-istituzionali dei governi, costituiscono, a nostro avviso, fattori rilevanti per la spiegazione delle differenze riscontrabili nelle diverse esperienze europee. Fattori che analizzeremo congiuntamente poiché è dalla loro interrelazione che emergono le rispettive potenzialità esplicative.

Come illustrato nel secondo paragrafo, i casi nazionali oggetto del nostro studio, rispetto al primo fattore, possono essere classificati a seconda che mantengano importanti prerogative di governo dell'istruzione a livello nazionale (Francia, Italia, Spagna e Svezia) o che deleghino tali prerogative a livelli inferiori (Gran Bretagna, Germania, Olanda e Belgio).

Una ulteriore distinzione che si può porre tra i paesi analizzati, e siamo al secondo fattore, è quella tra gli Stati caratterizzati da governi forti e Stati caratterizzati da governi deboli. Forza e debolezza che possiamo ricondurre alle più generali caratteristiche, rispettivamente, dei sistemi maggioritari e dei sistemi consensuali, secondo la nota distinzione di Arendt Lijphart (1984), e che deriva principalmente dalla più o meno netta sovrapposizione tra maggioranza e minoranza da un lato e governo e opposizione dall'altro (Cotta 1986), e dalla conseguente capacità del governo di portare a termine le proprie politiche (fattore legato alla maggiore o minore omogeneità della coalizione di governo), nonché dal grado di impermeabilità/permeabilità del governo stesso rispetto ai gruppi di pressione. Collocheremo così nella prima classe $i$ casi caratterizzati da elevata o media forza di governo (Francia, Gran Bretagna, Germania, Spagna, Svezia), nella seconda quelli caratterizzati da bassa forza di governo (Belgio, Olanda, Italia).

Se poniamo in relazione $\mathrm{i}$ casi nazionali classificati secondo il livello dell'unitarietà raggiunto in seguito alle riforme, con le due variabili relative all'accentramento delle istituzioni preposte al governo dei sistemi di istruzione e alla forza decisionale dei governi, otteniamo le tabelle 1 e 2 : 
che si colloca in un contesto di debole regolamentazione nazionale, che non frappone ostacoli alle innovazioni avviate a livello locale, e di assenza di potenziali poteri di veto reciproci tra le diverse autorità locali grazie alle particolari caratteristiche dei titoli di studio, privi di valore legale, e alle modalità di accesso all'istruzione universitaria, definite al livello dell'istruzione superiore, e non a quello dell'istruzione secondaria. Questo non significa affermare semplicemente che le riforme della scuola secondaria di carattere unitario sono state rese possibili sia da sistemi accentrati che da sistemi decentrati. Significa invece sostenere che esse sono state rese possibili sia dalla assunzione di importanti prerogative a livello nazionale, sia da una diffusa distribuzione di poteri a livello locale in assenza di veti provenienti dal livello nazionale, nonché di veti reciproci tra le numerose autorità locali. Dunque non un qualsiasi sistema decentrato si presenta come potenzialmente favorevole a riforme strutturali. In taluni casi, anzi, il decentramento può inibire le riforme, divenire per esse un ostacolo (Ocde 1995). È questo il caso della Germania, posta nella classe dei sistemi decentrati. Come abbiamo visto, nel caso tedesco lo spostamento verso il basso delle più importanti prerogative in materia scolastica si ferma a livello degli stati della federazione, tanto che si è parlato a questo proposito di un sistema multicentralizzato. Ogni decisione rilevante richiede l'accordo unanime dei Länder, ognuno dei quali possiede un elevato potere di veto. Nonché un più generale potere di ricatto nei confronti delle iniziative autonomamente prese all'interno dei singoli stati, in particolare attraverso l'arma del non riconoscimento dei titoli di studio rilasciati da scuole giudicate eccessivamente innovative e lontane dai parametri tradizionalmente riconosciuti nel paese. La particolare modalità di decentramento dei poteri in Germania inibisce inoltre, nel campo dell'istruzione, la possibilità di iniziative a un livello di governo più basso rispetto agli stati, iniziative che hanno invece avuto la possibilità di dispiegarsi nel caso inglese.

I due paesi che hanno introdotto solo limitate trasformazioni, Belgio e Olanda, sono posti anch'essi nella classe dei sistemi decentrati. Anche per questi casi l'impossibilità di introdurre riforme più incisive può essere, per lo meno parzialmente, ricondotta alla complessità e diffusione dei processi decisionali inerenti la materia scolastica. Anche per essi le differenze con il caso inglese sono evidenti. Le decisioni più importanti relative all'organizzazione del sistema scolastico non sono, infatti, de- 
mandate ai livelli locali, più prossimi agli istituti, e nemmeno agli istituti medesimi, bensì alle organizzazioni di vertice dei numerosi settori in cui sono suddivisi i sistemi di insegnamento dei due paesi, organizzazioni che, come nel caso dei Länder, possono esercitare poteri di veto.

Per quel che concerne il secondo fattore, le eccezioni all'ipotesi formulata sono rappresentate dall'Italia e dalla Germania. Nel caso italiano all'assenza del fattore governo forte corrisponde, infatti, la presenza di una importante riforma, quella della scuola media unica del 1962. Le ragioni di questa apparente devianza sono da ricercarsi, a nostro avviso, nelle specifiche dinamiche politiche proprie della fase durante la quale fu condotta in porto la riforma: l'avvio dell'esperienza del centro-sinistra (19621963). Una fase caratterizzata da una chiusura del processo decisionale di governo alle forze di opposizione (Pci), chiusura che sarebbe venuta meno negli anni successivi ed in particolare a partire dal 1968, e dalla volontà del partito di maggioranza relativa, la Democrazia cristiana, di raggiungere un accordo su obiettivi - nazionalizzazione dell'energia elettrica e scuola media unica - ai quali il partito socialista aveva condizionato la propria astensione al nuovo governo. Le particolari condizioni che hanno caratterizzato il periodo storico in cui è stata realizzata la riforma della scuola secondaria inferiore rendono così, almeno a noi pare, l'esito positivo di tale riforma più l'eccezione che non la regola nel più generale panorama della politica scolastica italiana, caratterizzata dall'assenza di decisioni rilevanti rispetto alle questioni cruciali dell'istruzione, come mostra la mancata attuazione della riforma della scuola secondaria superiore nonostante i reiterati tentativi di condurla in porto, avviati all'inizio degli anni settanta e non ancora conclusi.

Un'ulteriore eccezione potrebbe essere quella della Germania: governo forte e assenza di riforme incisive. Appare evidente che il fattore attinente le caratteristiche del governo, potenzialmente favorevole alle riforme, è stato inibito dalla particolare configurazione del sistema di governo dell'istruzione, multicentralizzato. Se, dunque, il livello di governo dell'istruzione e quello, più generale, del sistema politico non coincidono, la forza del secondo non necessariamente si riflette su quella del primo.

Per quanto concerne il caso inglese, si potrebbe supporre che la particolarità del processo che ha condotto alla creazione delle comprebensive schools renda non significativa la variabile forza del governo. Non va però sottovalutato, come ha notato il 
sociologo dell'educazione Benadusi (1989b, 269), il ruolo giocato dal governo laburista nell'affermazione definitiva di tale tipo di scuola, già largamente diffusa grazie agli interventi di numerose Local Education Authorities negli anni sessanta, anni in cui comincia timidamente a svilupparsi, nel settore scolastico, una più incisiva iniziativa governativa.

I casi belga e olandese, infine, rientrano nella classe dei governi deboli (ricordiamo che forza e debolezza riguardano non la stabilità dei governi quanto la loro capacità decisionale). Non avrebbe potuto essere diversamente, poiché l'accomodamento che è stato raggiunto dalle forze politiche nel campo dell'istruzione costituisce un elemento, tutt'altro che irrilevante, del più ampio accordo consociativo tra le medesime (Lijphart 1968; 1977) che è rimasto stabile fino agli anni sessanta ma che ancora oggi non può dirsi completamente esaurito - Lijphart (1988) collocava alla fine degli anni ottanta tali paesi nella più generale classe dei paesi consensuali. Forza del governo centrale e configurazione del governo del sistema scolastico sono dunque, in questi due specifici casi, variabili non indipendenti ma strettamente correlate e nella loro correlazione sfavorevoli all'innovazione nel campo dell'istruzione.

Tornando alle due ipotesi iniziali, appare evidente come esse vadano ulteriormente specificate. L'accentramento del governo del sistema scolastico e la sua collocazione al livello nazionale, può essere considerato un fattore potenzialmente favorevole a importanti innovazioni solo se accompagnato dalla contestuale presenza di un governo con elevata o media capacità decisionale, come mostra il fallimento dei più che trentennali tentativi di riformare la scuola secondaria superiore in Italia. Al tempo stesso la presenza di un governo forte non costituisce di per sé fattore sufficiente, dal momento che la sua azione può essere inibita da una particolare configurazione del governo dell'istruzione, come quella definita multicentralizzata (caso tedesco). Inoltre è necessario aggiungere una ulteriore ipotesi alle precedenti, e cioè che l'innovazione possa essere favorita anche in particolari sistemi decentrati, definibili di pluralismo diffuso (caso inglese) e come tali differenti dai sistemi multicentralizzati (espressione coniata per il caso tedesco e a nostro avviso utilizzabile anche per quelli belga e olandese, nonostante la centralizzazione interna alle istanze intermedie negli ultimi due casi sia notevolmente inferiore), che invece costituiscono un freno per le trasformazioni più incisive. 
Nella letteratura un importante ruolo per l'attuazione di riforme in senso unitario è stato attribuito anche alla presenza al governo, per un lungo periodo, dei partiti socialisti. Ad esempio, lo studioso Sixten Marklund ha individuato tra i fattori esplicativi delle ampie e incisive riforme realizzate in Svezia il progressismo socio-politico, caratterizzato dall'obiettivo dell'uguaglianza delle opportunità educative, espressosi a partire dagli anni cinquanta e sessanta attraverso i governi guidati dal partito socialdemocratico, che ne aveva assunto gli scopi, inserendoli all'interno del suo programma di welfare state (Marklund 1986, 34).

Tale fattore ha indubbiamente favorito il raggiungimento dell'obiettivo di una scuola unitaria in Svezia e in Spagna, ma nel generale quadro europeo non può essere considerato necessario, come dimostra il caso della Francia, dove la riforma del collège è stata opera di governi di centro-destra. Esso tutt'al più può contribuire a spiegare la più ampia realizzazione dell'obiettivo unitario all'interno della classe di casi che hanno comunque attuato riforme in questa direzione (Svezia e Spagna vs. Francia). Al tempo stesso l'influenza del ruolo dei partiti socialdemocratici può sussistere anche in assenza di una loro lunga permanenza al governo. Un esempio è dato dal caso inglese dove il partito laburista ha fornito, grazie all'adesione all'obiettivo dell'unitarietà della scuola secondaria all'inizio degli anni cinquanta, un impulso di carattere nazionale alle politiche delle Lea laburiste e un più generale input all'adeguamento del sistema al modello comprensivo con la circolare del 1965 del ministro laburista Anthony Crosland. Un secondo esempio è inoltre fornito dal caso italiano, dove la presenza del partito socialista, appena entrato a far parte, anche se indirettamente, della coalizione di governo, ha costituito un fattore determinante per l'attuazione di una riforma della scuola secondaria inferiore di tipo unitario e indifferenziato.

Appare invece evidente come la semplice presenza del partito socialista al governo, in assenza di un contesto istituzionale che gli fornisca gli strumenti per operare, non costituisca di per sé fattore in grado di condurre a trasformazioni in senso unitario dell'ordinamento scolastico. Come mostra il caso tedesco, dove il particolare assetto del governo dell'istruzione non ha reso possibile l'attuazione di politiche radicalmente innovative da parte del partito socialdemocratico, al governo dal 1969 al 1982. 
Per quanto riguarda l'ultimo fattore preso in considerazione da Benadusi, vale a dire le ideologie e le retoriche educazionali socialmente diffuse, va innanzitutto rilevato che esse spiegano sia la generale propensione verso le riforme del livello secondario in Europa (vedi la diffusione di istanze egualitarie e di democratizzazione negli anni sessanta), sia, in concomitanza con altri fattori, le differenze nei processi e negli esiti riformatori, nella misura in cui assumono caratteri specifici nei diversi ambiti nazionali. È lo stesso Benadusi, che tanta importanza attribuisce a questo fattore, che riconosce, però, come esso stesso sia riconducibile «sia al diverso impatto della variabile economica sia a residui di carattere storico, istituzionale e ideologico» (Benadusi 1989b, 223). Proprio su questi residui vorremmo soffermarci in conclusione. Essi altro non sono che fattori strutturali e cognitivi (Lanzalaco 1995) istituzionalizzatisi durante i processi che hanno condotto alla formazione dei sistemi di insegnamento e al loro inserimento nel più complesso sistema politico e sociale. Fattori quali le strutture dei sistemi di istruzione (intese come strutture dell'amministrazione ma anche degli ordinamenti), gli atteggiamenti dei diversi gruppi sociali rispetto al sistema educativo e agli obiettivi ad esso imputabili, il collegamento tra ruoli nella società e competenze formalmente definite dai diversi percorsi del sistema di istruzione e la correlata concezione del rapporto tra attività intellettuale e attività manuale. Fattori che laddove si sono cristallizzati attorno ad un sistema tripartito di istruzione hanno frenato le riforme in senso comprensivo. E questo il caso della Germania, tradizionalmente caratterizzata da uno stretto legame tra tipi e livelli di istruzione e ruolo professionale, nel contesto di una netta preminenza della cultura umanistica classica rispetto a quella scientifica e tecnica e di una rigida separazione tra attività intellettuali e attività esecutive. A questo proposito è stato infatti osservato come tra i fattori che hanno inibito l'innovazione dell'ordinamento scolastico tedesco, vi sia l'immagine tradizionalista della società, fortemente radicata nella cultura tedesca, e la concezione idealista del ruolo delle élite culturali (Max-Planck-Institute 1991, 17 19). Una situazione molto diversa da quella della Svezia dove già dalla fine del XIX secolo si era manifestata la tendenza ad un allontanamento dalle tradizioni e un'accentuazione degli aspetti più tecnici dell'istruzione. Il permanere di una concezione tradizionale della società e il riconoscimento diffuso della legittimità di un sistema di istruzione altamente selettivo finalizza- 
to alla creazione della classe dirigente, sono fattori che possono fornire anche un ulteriore tassello per la spiegazione del minor livello di unitarietà raggiunto nella école unique francese, che, come abbiamo visto, mantiene la presenza di opzioni alternative a partire già dal terzo anno (tredicesimo anno di età) del percorso secondario inferiore.

Se, dunque, dal lavoro qui presentato emerge l'importanza di fattori inerenti le caratteristiche politico-istituzionali dei sistemi politici e le più specifiche caratteristiche dell'amministrazione del sistema scolastico, da quanto illustrato in conclusione appare evidente che essi non possono esaurire una soddisfacente spiegazione degli esiti delle politiche scolastiche, esiti che non possono che essere imputati a complessi intrecci di fattori, dei quali altro non è possibile fare che individuare il potenziale carattere più o meno favorevole alle riforme e la reciproca capacità di rafforzare o indebolire tale carattere.

\section{Riferimenti bibliografici}

Alber, J. (1987), Dalla carità allo Stato sociale, Bologna, Il Mulino. Archer, M. (1984), Social Origins of Educational Systems, London, Sage.

Benadusi, L. (a cura di) (1989a), La non decisione politica. La scuola secondaria tra riforma e non riforma. Il caso italiano a confronto con altre esperienze europee, Firenze, La Nuova Italia.

- (1989b), La politica scolastica: processi di riforma a confronto, in Moscati (1989).

Biblioteca di Documentazione Pedagogica (Bdp) (1994), Le autonomie scolastiche: una lettura in chiave europea.

Boucher, L. (1982), Traditions and Change in Swedish Education, Oxford, Pergamon Press.

Cotta, M. (1986), Governi, in G. Pasquino (a cura di), Manuale di scienza della politica, Bologna, Il Mulino.

Di Francia, C. e P. Luggeri (1991), La scuola nell'Europa comunitaria, Latina, Edizioni Bucalo.

Draghicchio, E. (1980), I sistemi scolastici in Europa. Scuola dell'infanzia e dell'obbligo. Strutture e problemi, Firenze, Nuova Guaraldi.

Enderwitz, H. (1963), Two German Education Schemes: The Rabmenplan and the Bremerplan, in «Comparative Education Review», VII, n. 1, pp. 47-50.

Fanelli, S. (1989), Francia. Una riforma tra progetto e attuazione, in Benadusi (1989a). 
Fenwick, K. e P. McBride (1981), The Government of Education in Britain, Oxford, Martin Robertson.

Franchi, G. e T. Segantini (1994), La scuola che non ho. Per una politica della piena scolarità, Firenze, La Nuova Italia.

Franchi, G. e M. Todeschini (a cura di) (1977), La riforma in Europa. Persistenza e innovazione nei sistemi scolastici e formativi di Svezia, Francia, Germania e Gran Bretagna, Milano, Isedi.

Gigante, M. (1988), L'amministrazione della scuola, Padova, Cedam.

Giunta La Spada, A. (1989), Sistemi scolastici e politiche educative, Roma, La Nuova Italia Scientifica.

Heidenheimer, A.J. (1974), The Politics of Educational Reforms: Explaining Different Outcomes of School Comprehensivization Attempts in Sweden and West Germany, in «Comparative Education Review», XVIII, n. 3, pp. 388-410.

- (1983), Education Policy, in Heidenheimer, Heclo e Adams (1983).

Heidenheimer, A.J., H. Heclo e C.T. Adams (1983), Comparative Public Policy, New York, St. Martin Press.

Husén, T. (1986), Why did Sweden go Comprehensive?, in «Oxford Review of Education», XII, n. 2, pp. 153-163.

Lanzalaco, L. (1995), Istituzioni, Organizzazione, Potere. Introduzione all'analisi istituzionale della politica, Roma, La Nuova Italia Scientifica.

Leclercq, J.M. (1993), L'enseignement obligatoire en Europe, Paris, La Documentation française.

Lijphart, A. (1968), The Politics of Accomodation. Pluralism and Democracy in the Netherlands, Berkeley-Los Angeles, University of California Press.

- (1977), Democracy in Plural Societies, New Haven, Yale University Press.

- (1984), Democracies. Pattern of Majoritarian and Consensus Government in Twenty-One Countries, New Haven, Yale University Press (trad. it. Le democrazie contemporanee, Bologna, Il Mulino, 1988).

Marklund, S. (1986), La democratizzazione dell'istruzione in Svezia, in «Scuola democratica», IX, n. 1, pp. 34-39.

Max-Planck-Institute für Bildungsforschung (1979), Das Bildungswesen in der Bundesrepublik Deutschland, Reinbek bei Hamburg, Rowohlt Taschenbuch Verlag $\mathrm{GmbH}$, trad. francese aggiornata, Traditions et transformations. Le système d'éducation en République féderale d'Allemagne, Paris, Economica, 1991.

Morini, E. (1977), Gran Bretagna, Autonomia, sistema misto e gradualismo, in Franchi e Todeschini (1977).

Moscati, R. (a cura di) (1989), La Sociologia dell'educazione in Italia, Bologna, Zanichelli.

Ocde (1981), Examens des politiques nationales d'éducation. Les réformes de l'enseignement en Suède, Paris, Ocde. 
- (1991), Examens des politiques nationales d'éducation. Pays-Bas, Paris, Ocde.

- (1992), Examens des politiques nationales d'éducation. Belgique, Paris, Ocde.

- (1994), La formation professionnelle aux Pays-Bas: réforme et innovations, Paris, Ocde.

- (1995), Les processus de décision dans 14 systèmes éducatifs de l'Ocde, Paris, Ocde.

- (1996), Examens des politiques nationales d'éducation. France, Paris, Ocde.

Pasini, R. (1977), Repubblica Federale Tedesca: vecchi strumenti e nuovi problemi, in Franchi e Todeschini (1977).

Prost, A. (1968), Histoire de l'enseignement en France. 1800-1967, Paris, Armand Colin.

- (1992), Éducation, société et politique. Une bistoire de l'enseignement en France, Paris, Seuil.

Rescalli, G. (1996), Il cambiamento nei sistemi educativi. Processi di riforma e modelli europei a confronto, Firenze, La Nuova Italia.

Trivellato, P. (1984), La politica della scuola, in U. Ascoli (a cura di), Welfare State all' italiana, Bari, Laterza.

- (1989), Sistema scolastico e welfare state, in Moscati (1989).

Van Lutsemburg Maas, J. (1964), The «Mammouth Law» Reform in Dutch Education, in «Comparative Education Review», n. 1, pp. 279-285.

Zocchi, P. (1989), Svezia. Una riforma in movimento, in Benadusi (1989a). 